Published in final edited form as:

Clin Gerontol. 2016 ; 39(3): 190-209. doi:10.1080/07317115.2016.1151968.

\title{
"We can't provide season tickets to the opera": Staff perceptions of providing preference based person centered care
}

\author{
Katherine M. Abbott, PhD, MGS [Assistant Professor of Gerontology, Scripps Research \\ Fellow], \\ Department of Sociology and Gerontology, Miami University, 398 Upham Hall, Oxford, OH 45056, \\ abbottkm@miamioh.edu, 215-813-8131
}

Allison R. Heid, PhD, and

Rowan University School of Osteopathic Medicine, New Jersey Institute for Successful Aging, 42

E. Laurel Road, UDP Suite 2300, Stratford, NJ, heid@rowan.edu, 856-566-44594

Kimberly Van Haitsma, PhD [Associate Professor, Director, Program for Person Centered Living Systems of Care]

College of Nursing, The Pennsylvania State University, 201 HHD East, University Park, PA 16802, ksv110@psu.edu

\section{Abstract}

Knowledge of a nursing home resident's everyday living preferences provides the foundation for ongoing individualized care planning.

Objective-The purpose of this study is to identify nursing home (NH) staff perceptions of facilitators and barriers to learning about and meeting residents' preferences and reasons why staff feel residents change their minds about preferences.

Methods-Focus group sessions and interviews were conducted with $36 \mathrm{NH}$ staff members working in a facility that has been actively assessing resident preferences for five years.

Results-Thematic codes classifying facilitators, barriers, and dependencies were identified. Staff shared ways they are able to help meet residents' preferences as well as barriers to fulfilling resident preferences through their own behaviors, facility characteristics, the social environment, and resident characteristics. In addition, staff believe that residents change their minds about important preferences 'depending on' several factors including; global environmental characteristics, social environment, resident characteristics, and general staff perceptions.

Conclusions-This work identifies key facilitators and barriers to consider when implementing quality improvement efforts designed to improve the person-centered nature of care in nursing homes and is intended to further inform the culture change movement, which aims to transform NHs by empowering staff and delivering person-centered care.

\section{Introduction and Rationale}

The Affordable Care Act (US Department of Health and Human Services, 2014) places the individual at the center of the health care delivery process (Kietzman, 2012). Individuals are seen as key participants in shaping the goals and outcomes of their care. This policy is in line with the growing consensus among long-term care stakeholders that person-centered 
care is an important component of quality (Center for Excellence in Assisted Living, 2010; Centers for Medicare and Medicaid Services [CMS], 2006, 2011a, 2011b). It is consistent with the goals of the Centers for Medicare and Medicaid (CMS), in which the 8th Scope of Work endorses culture change efforts by directing state Quality Improvement Organizations (QIOs) to work with Nursing Homes (NHs) to "improve organizational culture" to honor the individual (Centers for Medicare and Medicaid Services, 2009). And recently, CMS proposed enhanced person-centered care processes during the 2015 White House Conference on Aging (LeadingAge, 2015). Such a focus on the individual further aligns with new federal regulations directed toward incorporating the assessment of personcentered activities into NH survey procedures (Hamilton, 2009).

Nursing homes (NH) are embracing person-centered care (PCC), an approach that emphasizes "knowing the person" and honoring each resident's preferences. A recent expert panel on PCC released this definition:

"'Person-centered care" "means that individuals' values and preferences are elicited and, once expressed, guide all aspects of their health care, supporting their realistic health and life goals." (American Geriatrics Society, 2015, p. 2).

Thus, one key element of individualized, person-centered care (PCC) is customizing care processes to fit the unique needs and preferences of each resident (Edvardsson et al., 2014). The literature has strongly supported the beneficial outcomes associated with tailoring care in nursing homes (e.g., Cvengros, 2009; Jahng, Martin, Golin, \& DiMatteo, 2005). Vallerand and O'Connor (1989) reported that nursing home residents who experience greater autonomy in self-care, religious, interpersonal and recreational activities report lower depression - as well as higher self-esteem, life satisfaction, meaning in life, general health and psychological adjustment. Meanwhile, Kasser and Ryan (1999) found that greater selfdetermination was associated with higher levels of psychological adjustment in nursing home residents. These findings partnered with the shift in regulatory focus to the individual underscore the critical importance of nurturing the needs of older adults through preferencebased care in NHs.

As such, the focus of recent research has been directed toward developing tools that enable long-term care providers to efficiently customize care. One approach is to measure preferences for care delivery and aspects of everyday living (Van Haitsma et al., 2012; Curyto, Van Haitsma, Towsley, in press). This strategy honors the experiences and continuity of likes and dislikes that individuals have developed over a lifetime (Brooker, 2007). In addition, it empowers residents, helping them to maximize their potential for retaining relationships, capabilities, interests and skills by acknowledging what they prefer in the context of their strengths and needs (Menne, Johnson, Whitlach, \& Schwartz, 2012). Personcentered approaches have been found to empower direct care staff and facilitate delivery of individualized care (Caspar, O'Rourke, \& Gutman, 2009), as well as improve job satisfaction (Edvardsson, Fetherstonhaugh, McAuliffe, Nay, \& Chenco, 2011).

Since 2010, NH facilities have been assessing 16 preferences annually through Section F of the MDS 3.0 (Saliba \& Buchanan, 2009; Housen, Shannon, Simon, Edelen, Cadogan, Buchanan, Saliba, 2009). Examples of these preferences include asking the resident to rate 
how important it is to choose what clothes to wear, choosing one's own bedtime, and/or listening to preferred music. The MDS requires input from multiple personnel to assess care outcomes for NH residents (therapy, social work, and clinical care staff). MDS coordinators, who are typically nurses, are responsible for completing the MDS assessment (Straker \& Bailor, 2008). In 2013, Advancing Excellence in America's Nursing Homes, a campaign to advance the quality of care in NHs, released a PCC toolkit that took the MDS preference assessment one step further to look at preference congruence (Van Haitsma et al., 2014). Preference congruence is an indicator of how well preferences align with care delivered and was measured through the reports of satisfaction by residents. Facilities are using the PCC toolkit to track preference congruence and are discussing ways to better align care with resident preferences during care planning meetings. However, while some advancements have been made in providing care that is more person centered, uptake of the implementation of PCC has been slow (Grabowski et al., 2014).

Prior work in this area highlights constraints of the institutional culture on allowing staff flexibility in care (Ulsperger \& Knottneurs, 2011; Persson \& Wästerfors, 2008). In addition, even in organizations with PCC supportive cultures there is a tendency for direct care workers to revert to care practices that are traditionally focused on tasks related to 'bed and body work' (Doyle \& Rubinstein, 2013). Communication breakdowns, time, and inadequate staffing are viewed by staff as major barriers to providing PCC (Kolanowski, Van Haitsma, Penrod, Hill, \& Yevchak, 2015). Staff believe that 'word of mouth' is the most efficient and reliable way to communicate knowledge about residents to one another because reading charts is too time consuming (Kolanowski et al., 2015). However, preferences for communicating PCC information by 'word of mouth' sparks concerns for determining the accuracy of the information as well as how information is shared consistently across disciplines and shifts (Kolanowski et al., 2015).

There is still much to learn from the perspective of staff members regarding their perceptions of facilitators and barriers they encounter in their day-to-day work in honoring resident preferences. As key stakeholders in delivering individualized, preference-based care, understanding the specific biases and/or interpretations of direct care workers is critical. The culture change movement has focused on empowering direct care workers. Staff have been called upon to have consistent assignments, respond to resident needs, offer choices, and be flexible, but there has been less of a focus on incorporating their views regarding how they go about providing preference-based person centered care (Koren, 2010). The person centered initiative initially bypassed staff input due to perceived conflicts of interest; however, direct care staff are critical in facilitating the delivery of such care.

Drawing upon the experience of a range of providers (e.g., social work, dietary, nursing, therapy) who have been actively working to incorporate resident preference information into practice using The Preferences for Everyday Living Inventory (PELI; Van Haitsma et al., 2012) for five years at a five-star 324 bed nursing facility, we sought to better understand the staff's perspective on delivering preference-based care to residents. The PELI has been administered by research staff and recreation therapists and asks residents to rate the importance of their preferences on 72-items in 5 domains: social contact, leisure and growth activities, diversionary activities, self-dominion, and enlisting others in care. Results are 
documented in each resident's medical record. All staff members are aware of the process. The purpose of this study was to identify: (1) main ideas of what staff think of the preference questions (i.e., general reactions), (2) how staff feel they help meet residents' preferences (i.e., facilitators), (3) barriers staff encounter when trying to meet residents' preferences (i.e., barriers), and (4) reasons staff perceive residents change their mind about preferences (i.e., dependencies). This work is intended to further inform the culture change movement in long-term care which aims to radically transform the nation's NHs by empowering staff and delivering person-centered care (Rahman \& Schnelle, 2008). This research is important to better understand the implementation issues in the movement away from a narrowly defined "medical" model toward a more holistic, bio-psycho-social model of care.

\section{Methods}

\section{Sample recruitment}

We utilized a convenience sample of 36 staff members from a 324 bed long-term care skilled nursing facility ( 270 long term beds and 54 short term rehab beds). The facility was chosen due to the fact that all staff having daily contact with residents had been involved in assessing and attempting to meet resident preferences for five years. This is not a common practice among nursing home facilities and we wanted to ask staff for their reflections on assessing and providing preference-based care because of their unique experiences. The authors were affiliated with a research institute that was embedded within the chosen facility. Eligible staff members were required to work at the facility and have direct contact with residents on a regular basis, be English speaking, and aged 18 or over. Staff needed to have permission from their respective supervisor to participate. Staff were recruited from Social Work, Recreation Therapy, Dietary, Certified Nursing Assistant (CNA) Morning Shift, CNA Evening Shift, and Nursing to gather a breadth of perspectives. Participants' discipline, race, and gender were recorded by the focus group moderator. See Table 1 for sample descriptive characteristics.

\section{Procedures and Analysis}

Three different procedures were followed to collect data based upon staff availability and schedule. Four in-person focus groups were conducted, 4 open ended questionnaires were completed, and one individual interview was completed. More specifically, full focus groups were not conducted with Social Work due to scheduling demands. Instead, Social Work staff $(n=4)$ voluntarily responded to the open-ended questions electronically by email. In addition, the Dietary department had a limited number of staff dieticians that could participate in a focus group. As a result, we completed one, in-person interview with a member of the dietary staff referred by the department head.

Participants were asked to respond to 4 broad questions: (1) What do you think about the preference items we are asking; (2) Describe an example of how you feel you have helped to do what a resident likes or prefers; (3) Describe an example of when you were not able to do what a resident prefers; and (4) Describe a time when a resident changed his or her mind about the importance of a given preference. In regard to this final question, prior work 
recognizes that $\mathrm{NH}$ residents' preferences may change or shift due to contextual factors (Heid et al., 2014), as a result we recognized the potential for staff to also see a "shift" in importance of a resident's preference as impacting their ability to provide preference-based care. Thus, staff perceptions on contextual dependencies impacting residents' preferences were also assessed. Appropriate follow-up questions were used to further probe for staff experiences with each question. Focus groups/interviews lasted on average 40 minutes.

All data were compiled by question and de-identified. For the in-person interview and the inperson focus groups, comprehensive field notes were taken during the interviews to document main ideas that were expressed (Stewart, 2006). To demonstrate rigor, interviews were audio recorded, transcribed and listened to for accuracy. To build a preliminary understanding of staff perceptions, all possible participants approved by supervisors were recruited.

Informed by content analysis strategies of Graneheim and Lundman (2004), data were read by two members of the research team independently, including the second author, to identify common categories or domains of responses. Codes were developed that captured each unique idea, or the manifest content (Graneheim \& Lundman, 2004). The data were coded utilizing each response given by a participant as a unit of analysis for the group interviews (i.e., for each participant's mention of an idea) and each unique thought following an interviewer prompt for individual interviews. Coded instances were counted to develop a sense of magnitude for each phenomenon (Wilkinson, 2011). Thematic codes started broad and were subsequently refined as factors were identified. The two Research Assistants met regularly to flesh out the application of the coding scheme; discrepancies were resolved by a team based consensus approach. A codebook was used to assure consistent application of codes.

A comment was coded as both a dependency and a barrier if it made reference to both a situation that would change the resident's preference importance rating and a situation that would restrict preference fulfillment. Similarly a comment was coded as both a dependency and a facilitator if it made reference to both a situation that would change the resident's preference importance rating and a situation that would enable preference fulfillment. A barrier or facilitator code did not indicate that participants would change their minds in any way around the preference if the barrier was removed.

\section{Results}

Focus Group sessions and interviews were conducted with 36 nursing home staff members representing social work $(n=4)$, dietary $(n=1)$, nursing $(n=6)$, recreation therapy $(n=8)$, and direct care (first shift $n=9$; second shift $n=8$ ) (See Table 1). Staff revealed several key findings regarding their perceptions. Verbatim quotations of themes illustrated most frequently are presented in this section.

\section{Significance and Methods of Discovering Preferences}

General reactions fell into two categories: 1) why it was important to ask about preferences and 2) the method by which staff discovered a preference. Staff reflected upon why it was 
important to ask nursing home residents' their preferences, articulating five primary reasons: to honor residents' rights, to provide better care, to promote or support choice or control, to ensure resident satisfaction with care, and because it is a normal part of the human experience and should be done. Two examples of the most frequently mentioned reasons for why it was important to ask about preferences included the following.

If I were here, and I were coming in to live, I would want to be able to choose what clothes to wear and what time I go to bed and I wouldn't want to listen to music that someone else is choosing, so that essentially is it. I think everyone has strong feelings about how they want to live their day to day life and they should really have the option to continue. (Importance of choice/control)

It's really no different than what my preferences are either. You know I think when they come in here we sometimes forget that they still have a life and we just, it gets so busy and it's not putting down the staff, it's easier just to go in and get your stuff and start providing care instead of asking them what they want. We forget they were something or somebody prior to coming here; they are not just old people waiting to their last day. (Importance of normalcy)

Staff also shared the methods by which they learn about resident preferences. They learned about residents' preferences through: formal organizational communication channels (i.e., software programs, assignment sheets, care planning processes, surveys, or committees), from other people (i.e., family members, the interdisciplinary staff, or other residents), and from residents themselves. When learning from the residents themselves, staff reflected on both direct ways of garnering resident self-report (i.e., asking the resident, listening to the resident, or the resident self-advocating), as well as ways of learning about their residents' preferences through care delivery (i.e., seeing residents' personal belongings, building a relationship, trial and error, interacting with the resident, getting to know the resident, or observing the resident) or residents' past history.

You ask them. Or like if someone has dementia or something you can tell by their reactions what they want and don't want. So like I said if you take care of them every day you ask them what they like and what they don't like and you build a relationship and you go from there. (Learn from Resident (ask))

If they come in not talking, you know, like end stage, then you communicate with the family or POA to give us their feedback of their likes and your dislikes to let us know your everyday routine so we can help take care of them. (Learn through Others)

Yea, eye contact, it kind of like depends on that person too, like if someone is a very introverted person, is going to be engaged in that group more passively, but you can still tell from their body language that they are enjoying the group or involved in the group. (Learn from Resident (observe))

Staff members see the value in learning about resident preferences and believe there is a benefit to the process. Staff have intuitively discovered multiple ways of collecting preference information from residents, other staff members, and through observation. Direct care staff are in a unique position to observe preferences and their knowledge and 
experiences can be leveraged by administrators when building PCC practice initiatives. See Table 2 for examples of all ways staff learned about preferences.

\section{Facilitators of preference fulfillment}

Interviewed staff also shared ways in which they were able to help meet residents' preferences. Facilitators included staff behaviors (i.e., staff accommodating the preference, offering an opportunity or choice, adapting the activity to meet a resident's needs/abilities, advocating for the resident, anticipating a resident's needs, making oneself available to address residents' preferences, educating residents or family, or gaining access to resources for the resident), facility characteristics (i.e., use of software, staff schedules, facility values, or physical facility environment), the social environment (i.e., family support, staff relationships, relationships with other residents, respect, supports from the community, or support from the interdisciplinary care team), and resident characteristics (i.e., health/ functional status change; see Table 3).

Some of them [preferences] are not possible here. We try to satisfy preferences but we can't provide season tickets to the opera kind of thing. (Staff Behaviors)

Do rounds, when we come in, we talk to them. "Oh, Mrs. Such and such, she had a bad day today, or she didn't want this done today, she wanted to have this done today. Or she might not be in a good mood today so she might just want to stay in her room". You just give the next person information about them. (Social Environment)

Staff recognize that there are many factors that support their ability to provide preferencebased care. The two most highly mentioned facilitators were staff providing or suggesting an opportunity for meeting a resident preference and garnering support from other staff members (See Table 3). Staff members are one of the most important, if not the most important element, to facilitating preference-based care. An environment where staff can work together in interdisciplinary teams to support resident preferences may prove critical in advancing PCC practice.

\section{Barriers to preference fulfillment}

Staff also reflected on barriers they encountered in trying to fulfill a person's preferences. These barriers included within-person characteristics of the staff (i.e., perceived lack of accuracy by staff of resident preferences, negative staff perceptions of residents, lack of control felt by staff, or lack of perceived ability to complete preferences in a timely manner), facility characteristics (i.e., facility policy, lack of facility resources, facility scheduling, the community living environment, staff overload, time constraints, staffing ratios, financial resources, government policy, or safety concerns), factors related to the social environment (i.e., perceived lack of resident networks, negative social contagion, family involvement, negative resident relationships, generational gaps, lack of support from administration or interdisciplinary team, lack of respect for staff, or staff feeling threatened by sanctions or loss of job), and resident characteristics (i.e., behaviors, persistence in ideas, different interests, mood, personal physical health, mental health, personal resources, personal schedule, functional ability, sensory ability, status change, cognitive ability, or competency). 
I think a lot of it is education too of residents and families and the understanding of what community living is all about. And that it may be okay for your loved one but there may be a risk to somebody that is demented that wanders into rooms and try to explain and educate why decisions are made and plus we are under the guides of Department of Health. I think in a lot of ways that is a big thing, for people that want to have fans or humidifies, or heating pads or the mattresses or the refrigerators in the room. [CHIMING IN FROM OTHERS, "heating pads", "curling irons"]. And everything can be, we have to follow the higher than us, which is the Department of Health. A lot of time that is where the regulations come into play. Some understand better than others. (Facility Characteristics: Policy \& Regulation)

If you're incontinent you can't go in bath. If usually you are a person that would get a bath, but now you are incontinent. Then that status change, now you are incontinent, so you are not able to go in the bath any more. So now we have to change to a shower where you sit. .... And, just like it breaks their heart it breaks our hearts too. Because we are knowing the fact that we can't meet your needs, but there is no way around it. It is difficult both ways. (Facility Characteristic: Facility Policy)

It is just kind of trying to meet the preferences of all 324 people and sometimes their families are involved in that too. So it becomes a very large challenge to do that. (Facility characteristic)

I think sometimes family can be a barrier. Like sometimes they want their mother or father to be up and out of bed. And sometimes the resident or patient doesn't want to. And sometimes they over step that bounds of listening to their mom or dad if they are awake, alert, and oriented there is no reason they cannot make decisions for themselves. And sometimes families are too pushy and it's not for the good of the mom or dad, it is actually doing more harm than good. (Social Environment)

I think every day people experience the changes their residents go through. Every day you come in sometimes it is something different from yesterday, every day you come in, "okay what is going to happen today". You never know when you come in. (Resident Characteristics)

Staff enumerated far more barriers than facilitators to providing preference-based care with facility policy being the most frequently cited barrier (See Table 4). Facility regulations were cited as restricting the ability of staff to fulfill preferences or acted in opposition to resident rights. Opening up a dialogue between regulators and providers surrounding these barriers will be a critical step toward mitigating these major barriers in providing preference based care.

\section{Dependencies}

Last, staff reflected on reasons for why they think residents' may change their minds about the importance of their preferences, indicating that residents' preferences "depend on" many different factors, which can impact care delivery. These dependencies included global environment characteristics (i.e., the weather), the social environment (i.e., family 
relationships, relationships with other residents, or staff relationships), resident characteristics (i.e., personal physical health, mental health, mood, functional ability, sensory ability, perceived social acceptability, general changing of one's mind, envy, having the opportunity or choice to change one's mind, cognitive ability, competency, or interest) and one other general staff perception (i.e., that residents' are just like children).

Just because their preferences change. I'm not the same person I was 10 years ago you know what I mean. I might decide I want to do more, or not want to go to bingo because last time I didn't win enough quarters, I don't know. People change their mind. They say the only constant in life is change. It is part of the human element in my opinion. (Resident Characteristic)

You might find out much later that they have had a tiff with someone they are close with (Social Environment)

Staff members were keenly observant of times when residents changed their minds about an important preference. Memory loss was the most frequently cited reason by staff for why they perceived residents changed their mind (See Table 5). Staff who work with residents with dementia recognize that preferences are not static, but dynamic and in need of frequent assessment. As we found with residents (Heid, et al., 2014), staff perceived that residents also changed their minds due to relationships with other residents, their mood, envy, and level of interest which can challenge their efforts to meet a given resident's preference on a given day (See Table 5).

\section{Discussion}

This study helps to understand nursing home staff members' perceptions regarding the importance of resident preference assessment, with a particular focus on how they learn about residents' preferences and the facilitators, barriers, and dependencies related to providing preference-based, person centered care. This study allowed for the inclusion of the voice of the direct care provider, whose buy-in is critical in honoring residents' preferences. This initial descriptive work identifies a host of key factors, from the perspective of staff, which must be considered when implementing quality improvement efforts directed at improving the delivery of person-centered, preference-based care.

Overall, staff felt that understanding residents' preferences was "reasonable and the right thing to do". Staff learned about preferences directly from residents, family, other staff members, and through paying attention to residents' actions and behaviors. Staff with more developed observational skills may be able to more quickly adapt care to resident preferences; such skills can be a focus for hands-on staff training. Electronic medical records and care planning were formal organizational channels through which staff accessed information about resident preferences. However, these may not be the most reliable and efficient means for staff to obtain preference information based upon previous studies (Kolanowski et al., 2015). Therefore, organizations can encourage staff members to explore how they informally learn about resident preferences and develop a systematic way of conveying this information across all shifts and disciplines. One example is including direct care workers in care planning meetings or resident conferences on a regular basis. 
Communicating resident preferences and discussing successful strategies from multiple disciplines can assist in empowering direct care staff in providing more individualized care (Kolanowski et al., 2015).

Facilitators supporting staff in honoring resident preferences included staff behaviors to accommodate, adapt, advocate, and anticipate resident needs, wants, and preferences. The leadership values of the facility in supporting PCC as well as environmental changes to the building to deliver PCC were also identified as facilitators. The social environment, which encompassed family support, staff relationships, and resident relationships, facilitated the provision of preference based PCC as well as resident characteristics such as improved resident health. Supporting staff in being creative in brainstorming ways to meet residents' preferences can empower staff to be thought leaders in PCC quality improvement efforts within a facility.

However, as expected, staff perceived far more barriers than facilitators. Facility characteristics such as policies, resources, scheduling, staffing ratios, and safety prevented staff from fully honoring resident preferences. The social environment also functioned as a barrier when family preferences differed from resident preferences or when residents did not get along with one another. One opportunity facilities may have is to group residents with similar recreational and leisure preferences, even if resident physical and functional ability differs, in order to allow for a focus on offering preferred activities. Additionally, negative staff perceptions of residents, the lack of control felt by staff, and staff not being able to address a preference in a timely manner were also barriers that inhibited resident preference fulfillment. The biomedical model of task efficiency is an underlying force even in nursing homes who have adopted PCC as part of the culture (Doyle \& Rubinstein, 2013). Therefore, continuous conversations among direct care staff and supervisors/champions who can role model PCC practices is crucial for sustaining person-centered care (Kolanowski, 2015).

Staff also identified that behaviors of residents restrict preferences from being met. We argue that behaviors of wandering, anger, and resistance to care can be reflections of preferences not being met. In addition, resident interest, mood, physical and mental health all lead to difficulties in staff meeting preferences. Some staff perceived the resident's personal schedule as a barrier to meeting preferences, along with functional ability. Hearing, visual, cognitive, and functional impairments greatly restrict staff ability to fulfill preferences. It is in these situations that Certified Therapeutic Recreation Specialists (CTRS) can thoughtfully adapt activities to meet resident important preferences based upon physical, functional, and cognitive limitations (Van Haitsma et al., 2014).

Lastly, staff identified a series of reasons for why residents may change their minds about preferences. We have labeled these 'situational dependencies' that affect the staff's ability to deliver PCC because they create uncertainty. This uncertainty is met on a daily basis as staff are required to quickly assess not only resident characteristics that may have changed overnight, but contextual and relational characteristics that impact how important a preference will be to a resident at a given time. Staff discussed global characteristics, such as the weather changing if a resident wanted a preference fulfilled. Social environment dependencies were based upon the relationship between the resident and his/her family, 
other residents, and staff that made fulfilling some resident preferences 'dependent on' the people they were doing the activity with. The category with the most dependencies, according to staff, focused on resident characteristics. Staff felt that meeting resident preferences was difficult sometimes based upon such things as the residents' health, changing their minds, interest level, and cognitive ability. Training staff to be aware of such contextual dependencies is important to help staff recognize the many ways meeting a preference can 'depend upon' how a resident is feeling, who the activity is with, and of course, the weather.

Of interest, staff reports of barriers and dependencies paralleled residents' perspectives found in prior research (Bangerter et al., in press; Heid et al., 2014). We found that there were 14 similar barriers reported and 25 additional unique barriers discussed by staff. Some of the overlapping barriers included facility policies, resources, and schedule as well as resident characteristics such as interest, mood, health, and ability. We also found that there were 11 similar dependencies reported and 6 additional unique perceived dependencies discussed by staff. We found overlapping dependencies related to the weather and resident characteristics (e.g., health, mood, functional and sensory ability, and choice). Such results validate residents' perspectives, highlighting systematic barriers and contextual circumstances that affect the importance of residents' preferences and whether their preferences can be fulfilled. The similarities also can be a way of uniting residents and staff through common successes and frustrations, instead of focusing on an 'us vs. them' mentality (Tajfel, 1979).

As with all studies there are limitations to this research. The staff perspectives gathered were from a single five star facility located in the northeast United States that has been formally performing preference assessments for five years. Staff needed to have received permission from their respective supervisor to participate, which may have biased the sample of staff members who volunteered. Further results did not compare perceived barriers or facilitators across educational level. Additional work should explore staff members' perceptions within other facilities and larger samples to ensure saturation of results demonstrated here and examine factors that may influence differential perspectives in care. In addition, multiple methods of data collection were employed in order to work around staff schedules. Some perspectives were from individuals, while other perspectives grew out of group discussions (focus groups). Future research exploring staff perceptions on the facilitators, barriers, and dependencies of providing preference-based, PCC should seek additional staff perspectives, such as housekeeping supervisors or environmental services, and examine the role of interdisciplinary team approaches in addressing preferences in care. In addition, sharing these findings with stakeholder groups to identify key practice recommendations based on staff members' perspectives of residents' preferences is crucial. The development of clinical recommendations for addressing barriers and supporting facilitators of resident preferences will empower staff to continue to move toward more preference congruent care.

\section{Conclusion}

This work assists in developing an understanding about how to optimize delivery of preference-based, person-centered care through identifying the major facilitators and barriers 
at the facility, staff, and resident levels. Staff training and education can be tailored to develop facilitators and address barriers as care teams. Major facility policies that limit staff ability to provide preference congruent care can be explored and potentially revised. Negotiations of risk may be needed in order for residents to fulfill preferences despite possible safety concerns. Overall, we find that most of the staff members interviewed here strive to deliver preference based care to nursing home residents, yet important constraints remain.

\section{Acknowledgements}

We would like to thank Christina Duntzee, a research team member who worked diligently to collect this data and the staff members who participated in the project.

Funding: This work was made possible by funding from the Donaghue Foundation and an NINR grant (1R21NR011334: PI Van Haitsma).

\section{References}

The American Geriatrics Society Expert Panel on Person-Centered Care. 2015 Article first published online. doi: 10.1111/jgs.13866.

Bangerter L, Heid A, Abbott KM, Van Haitsma K. "Make me feel at ease and at home" Differential Care Preferences of Nursing Home Residents. The Gerontologist. in press.

Brooker, D. Person-Centered Dementia Care: Making Services Better. Jessica Kingsley; London: 2007.

Caspar S, O'Rourke N, Gutman GM. The differential influence of culture change models on long-term care staff empowerment and provision of individualized care. Canadian Journal on Aging. 2009; 28:165-175. doi:10.1017/S0714980809090138. [PubMed: 19860975]

Center for Excellence in Assisted Living. [June 1, 2015] Person-centered care in assisted living: An informational guide. 2010. from http://www.theceal.org/assets/PDF/Person-Centered\%20Care $\% 20 \mathrm{in} \% 20$ Assisted\%20Living.pdf

Centers for Medicare and Medicaid Services (CMS). Home healthcare quality initiatives: Overview. 2011a. Retrieved from https://www.cms.gov/homehealthqualityinits/

Centers for Medicare and Medicaid Services (CMS). Minimum data set (MDS) - version 3.0. Resident assessment and care screening all item listing. 2011b. Retrieved from http://www.cms.gov/ NursingHomeQualityInits/30NHQIMDS30TechnicalInformation.asp\#TopOfPage/

Centers for Medicare and Medicaid Services. Policy council document. Long-term care plan. Sep 28. 2006 Retrieved from http://www.cms.gov/medicaidgeninfo/downloads/ltcreformplan2006.pdf

Curyto K, VanHaitsma K, Towsley G. Cognitive Interviewing: Revising the Preferences for Everyday Living Inventory (PELI) for use in the Nursing Home. Research in Gerontological Nursing. in press.

Cvengros JA. Patient preference for and reports of provider behavior: Impact of symmetry on patient outcomes. Health Psychology. 2009; 28:660-667. doi:10.1037/a0016087. [PubMed: 19916633]

Doyle PJ, Rubinstein RL. Person-centered dementia care and the cultural matrix of othering. The Gerontologist. 2013; 54:52-963. doi:10.1093/geront/gnt081.

Edvardsson D, Varrailhon P, Edvardsson K. Promoting person-centeredness in long-term care: An exploratory study. Journal of Gerontological Nursing. 2014; 40:46-53. doi: 10.3928/00989134-20131028-03. [PubMed: 24219071]

Edvardsson D, Fetherstonhaugh D, McAuliffe L, Nay R, Chenco C. Job satisfaction amongst aged care staff: Exploring the influence of person-centered care provision. International Psychogeriatrics. 2011; 23:1205-1212. doi:10.1017/S1041610211000159. [PubMed: 21320379]

Grabowski DC, O'Malley AJ, Afendulis CC, Caudry DJ, Elliot A, Zimmerman S. Culture change and nursing home quality of care. The Gerontologist. 2014; 54(Suppl. 1):S35-S45. doi:10.1093/geront/ gnt143. [PubMed: 24443604] 
Graneheim UH, Lundman B. Qualitative content analysis in nursing research: Concepts, procedures and measure to achieve trustworthiness. Nurse Education Today. 2004; 24:105-112. doi:10.1016/ j.nedt.2003.10.001. [PubMed: 14769454]

Hamilton, T. Nursing Homes - Issuance of Revisions to Interpretive Guidance at Several Tags, as Part of Appendix PP, State Operations Manual (SOM), and Training Materials. Center for Medicaid and State Operations/Survey and Certification Group; 2009. http://www.cms.hhs.gov/ SurveyCertificationGenInfo/downloads/SCLetter09_31.pdf [July 14, 2015]

Heid AR, Eshraghi K, Duntzee C, Abbott K, Curyto K, Van Haitmsa K. It depends': Reasons why nursing home residents change their minds about care preferences. The Gerontologist. 2014 Advance online publication. doi: 10.1093/geront/gnu040.

Housen P, Shannon GR, Simon B, Edelen MO, Cadogan MP, Buchanan J, Saliba D. Why not just ask the resident? Journal of Gerontological Nursing. 2009; 35:40-49. doi: 10.3928/00989134-20091001-01. [PubMed: 19904856]

Jahng KH, Martin LR, Golin CE, DiMatteo MR. Preferences for medical collaboration: Patientphysician congruence and patient outcomes. Patient Education and Counseling. 2005; 57:308-314. doi:10.1016/j.pec.2004.08.006. [PubMed: 15893213]

Kasser V, Ryan R. The relation of psychological needs for autonomy and relatedness to vitality, wellbeing and mortality in a nursing home. Journal of Applied Social Psychology. 1999; 29(5):935954.

Kietzman K. Using a "person-centered" approach to improve care coordination: Opportunities emerging from the Affordable Care Act. Journal of Geriatric Care Management Journal. 2012; 22(2):13-19.

Kolanowski A, Van Haitsma K, Penrod J, Hill N, Yevchak A. "Wish we would have known that!" Communication Breakdown Impedes Person-Centered Care. The Gerontologist. 2015; 55(Suppl 1):S50-60. doi: 10.1093/geront/gnv014. [PubMed: 26055781]

Koren MJ. Person-centered care for nursing home residents: The culture-change movement. Health Affairs. 2010; 29(2):321-317.

Menne H, Johnson J, Whitlach C, Schwartz S. Activity preferences of persons with dementia. Activities, Adaptation and Aging. 2012; 36(3):195-213.

Persson T, Wästerfors D. Such trivial matters: How staff accounts for restrictions of residents' influence in nursing homes. Journal of Aging Studies. 2008; 23:1-11. doi: 10.1016/j.jaging. 2007.09.005.

Rahman AN, Schnelle JF. The Nursing Home Culture-Change Movement: Recent Past, Present, and Future Directions for Research. Gerontologist. 2008; 48(2):142-148. [PubMed: 18483426]

Saliba, D.; Buchanan, J. Report for Centers for Medicare and Medicaid Services. HHS; 2009. Development and validation of a revised nursing home assessment tool: MDS 3.0.. from https:// www.cms.gov/Medicare/Quality-Initiatives-Patient-Assessment-Instruments/ NursingHomeQualityInits/downloads/MDS30FinalReport.pdf [August 1, 2015]

Stewart, DW. Focus Groups:Theory and practice 2nd edition. SAGE Publications Ltd.; London, England: 2006. Analyzing focus group data.; p. 109-134.

Straker J, Bailer J. A review and characterization of the MDS process in nursing homes. Journal of Gerontological Nursing. 2008; 34(10):36-43. [PubMed: 18942538]

Tajfel H, Turner JC. An integrative theory of intergroup conflict. The social psychology of intergroup relations. 1979; 33:47.

Ulsperger, JS.; Knottneurs, JD. Elder care catastrophe: Rituals of abuse in nursing homes and what you can do about it. Paradigm Publishers; Boulder, CO.: 2011.

US Department of Health and Human Services. Read the Law: The Affordable Care Act, Section by Section. 2014. Retrieved from http://www.hhs.gov/healthcare/rights/law/

Vallerand RJ, O'Connor BP. Motivation in the elderly : A theoretical framework and some promising findings. Canadian Psychology/Psychologie Canadienne. 1989; 30(3):538-550. doi: 10.1037/ h0079828.

Van Haitsma K, Crespy S, Humes S, Elliot A, Mihelic A, Scott C, Abbott K. New toolkit to measure quality of person-centered care: development and pilot evaluation with nursing home communities. Journal of the American Medical Directors Association. 2014 
Van Haitsma K, Curyto K, Spector A, Towsley G, Kleban M, Carpenter B, Koren MJ. The preferences for everyday living inventory: Scale development and description of psychosocial preferences responses in community-dwelling elders. The Gerontologist. 2012; 53(4):582-595. doi:10.1093/ geront/gns102. [PubMed: 22936532]

Wilkinson, S. Analyzing focus group data.. In: Silverman, D., editor. Qualitative Research. 3rd Edition. SAGE Publications Ltd; London, England: 2011. p. 168-184. 


\section{Table 1}

Demographic characteristics of sample

\begin{tabular}{lc}
\hline Variable & Staff $(\boldsymbol{N}=\mathbf{3 6})$ \\
\hline Gender (Female) & $92 \%(33)$ \\
\hline Race & \\
$\quad$ African American & $50 \%(18)$ \\
Caucasian & $47 \%(17)$ \\
Other & $3 \%(1)$ \\
\hline Discipline & \\
Direct Care Worker (Morning 7am to 3pm) & $25 \%(9)$ \\
Direct Care Worker (Evening 3pm to 11pm) & $22 \%(8)$ \\
Nurse & $17 \%(6)$ \\
Dietician & $3 \%(1)$ \\
Recreation Therapist & $22 \%(8)$ \\
Social Worker & $12 \%(4)$ \\
\hline
\end{tabular}


Table 2

How Staff Learn about Residents’ Preferences

\begin{tabular}{|c|c|c|c|}
\hline Code Name & Theme Description & Groups $^{a}$ & Citations $b$ \\
\hline \multicolumn{4}{|c|}{ Learn Resident Preferences through Formal Organizational Communication Channels } \\
\hline Software & Software (i.e. Geri-Menu- Dining tray system); Electronic Medical Record system & 1 & 3 \\
\hline Assignment Paper & Learn from assignment paper in charts completed by each shift & 2 & 3 \\
\hline Care Planning & Through care planning & 2 & 2 \\
\hline Survey & Resident completes printed survey (i.e., admission assessment when arrive) & 4 & 6 \\
\hline Committee & Program to gather feedback as a group (i.e., focus groups) & 1 & 1 \\
\hline \multicolumn{4}{|c|}{ Learn Resident Preferences through Others } \\
\hline Family & Learn from family what resident likes/liked; Learn from Power of Attorney (POA) & 6 & 18 \\
\hline Interdisciplinary Team & Learn about resident preferences from other staff (informally or through rounds) & 5 & 10 \\
\hline Other Resident & Other resident advocates for a resident & 1 & 1 \\
\hline \multicolumn{4}{|c|}{ Learn Resident Preferences from Residents } \\
\hline \multicolumn{4}{|c|}{ From Self-report } \\
\hline Ask & Ask residents & 6 & 23 \\
\hline Listen & Listen to residents' perspectives; Resident talks about preference & 4 & 12 \\
\hline Advocate & Resident self-advocating & 2 & 4 \\
\hline \multicolumn{4}{|c|}{ From Care Experience } \\
\hline Personal Effects & Seeing what is in the resident's room; Pictures, knick knacks & 2 & 3 \\
\hline Relationship & Build a relationship with resident & 2 & 3 \\
\hline Trial and Error & Trial and error to figure out what resident likes & 2 & 4 \\
\hline Interact & Staff interact with residents; Being with them & 3 & 5 \\
\hline Know & Get to know residents/person, experience with them; Learn what can do & 5 & 14 \\
\hline Observe & Just watch the residents to see what like/dislike & 4 & 16 \\
\hline \multicolumn{4}{|c|}{ From Past Experience } \\
\hline History & Learn about past preferences & 5 & 11 \\
\hline
\end{tabular}

Note. $N=36$ staff members within 6 groups.

Indicates the number of groups (Dietary staff, Resident Care Assistants - Morning shift, Resident Care Assistants - Evening shift, Nursing staff, Recreation staff, and Social Workers) the theme presented for.

$b_{\text {Indicates the number of citations of each theme in the transcripts. }}$ 
Table 3

Facilitators that Support Staff in Honoring Residents' Preferences

\begin{tabular}{|c|c|c|c|}
\hline Code Name & Theme Description & Groups $^{a}$ & Citations $b$ \\
\hline \multicolumn{4}{|l|}{ Staff Behaviors as Facilitators } \\
\hline Accommodate & $\begin{array}{l}\text { Staff physically assisting or accommodating resident to meet needs/preference; } \\
\text { Staff workaround policy to meet preferences }\end{array}$ & 5 & 14 \\
\hline Opportunity & Staff provide/suggest an opportunity for resident preference & 5 & 18 \\
\hline Adapt & $\begin{array}{l}\text { Staff adapt activity or match activity to meet needs/abilities, functionally and } \\
\text { sensory; Offer hand over hand support or adapt own abilities to meet resident } \\
\text { needs }\end{array}$ & 3 & 10 \\
\hline Advocate & Staff advocate for residents & 1 & 4 \\
\hline Anticipate & Staff anticipate needs/wants/preferences & 1 & 2 \\
\hline Available & $\begin{array}{l}\text { Staff make self available to do request or be there to support residents in } \\
\text { preference }\end{array}$ & 4 & 4 \\
\hline Education & Staff teach resident or family what can do to meet preferences & 3 & 5 \\
\hline Resources & $\begin{array}{l}\text { Staff gather resources for the preference to be met; Help make resources } \\
\text { available }\end{array}$ & 4 & 4 \\
\hline \multicolumn{4}{|c|}{ Facility Characteristics as Facilitators } \\
\hline Software & Data systemhelps show staff preferences to meet them & 1 & 1 \\
\hline Staff Schedule & Nursing schedules support it & 1 & 1 \\
\hline Values & Values of facility supported philosophy, facility buy in & 2 & 2 \\
\hline Environment & Physical environmental changes to building to deliver type of care & 3 & 8 \\
\hline \multicolumn{4}{|l|}{ Social Environment as Facilitators } \\
\hline Family Support & Staff garner family support, family helps in some way to meet preference & 1 & 1 \\
\hline Contagion & $\begin{array}{l}\text { Social contagion (i.e., enthusiasm, positive group emotions) facilitates } \\
\text { preferences }\end{array}$ & 1 & 1 \\
\hline Staff Relationships & Staff building positive relations with resident; Consistent assignment & 3 & 4 \\
\hline Resident Relationships & Staff connect residents with other residents & 2 & 6 \\
\hline Respect & Staff respect resident wishes and respect each other to support preferences & 3 & 3 \\
\hline Community Support & Staff bring in support from community- in and out of facility & 1 & 1 \\
\hline Interdisciplinary Team Support & $\begin{array}{l}\text { Garner support from other staff (i.e., supervisors or other nurses/CNAs); } \\
\text { Teamwork approach; Communicating persons preferences/needs to others }\end{array}$ & 5 & 18 \\
\hline \multicolumn{4}{|c|}{ Resident Characteristics as Facilitators } \\
\hline Status Change & Improved resident health/functional condition helps meet resident preferences & 1 & 1 \\
\hline
\end{tabular}

Note. $N=36$ staff members within 6 groups.

Indicates the number of groups (Dietary staff, Resident Care Assistants - Morning shift, Resident Care Assistants - Evening shift, Nursing staff, Recreation staff, and Social Workers) the theme presented for.

$b_{\text {Indicates the number of citations of each theme in the transcripts. }}$ 
Table 4

Barriers that Prevent Staff from Honoring Residents' Preferences

\begin{tabular}{|c|c|c|c|}
\hline Code Name & Theme Description & Groups $^{a}$ & Citations $^{b}$ \\
\hline \multicolumn{4}{|c|}{ Within Person (Staff) Barriers } \\
\hline Accuracy & $\begin{array}{l}\text { Staff perception of concern of accuracy of preferences or match of stated to } \\
\text { real preferences }\end{array}$ & 4 & 6 \\
\hline Staff Perceptions & Negative staff perceptions of residents & 1 & 2 \\
\hline Control & Lack of control felt by staff & 1 & 1 \\
\hline Timeliness & $\begin{array}{l}\text { Staff not being able to address preferences to resident liking on resident's } \\
\text { timeline }\end{array}$ & 1 & 1 \\
\hline \multicolumn{4}{|c|}{ Facility Characteristics as Barriers } \\
\hline $\begin{array}{l}\text { Facility Policy \& } \\
\text { Regulations }\end{array}$ & $\begin{array}{l}\text { Facility policies and regulations restrict preference or act in opposition to } \\
\text { resident rights }\end{array}$ & 4 & 25 \\
\hline Facility Resources & $\begin{array}{l}\text { Staff cannot get things need to meet preferences at the facility level; Quality } \\
\text { of resources not what needed/liked by resident }\end{array}$ & 3 & 6 \\
\hline Facility Schedule ${ }^{* *}$ & Facility schedule restricts preference fulfillment & 3 & 4 \\
\hline Community Living & $\begin{array}{l}\text { Not like at home, it is a community living environment; Sheer number of } \\
\text { people serving and preferences to be met constrains preference fulfillment }\end{array}$ & 3 & 4 \\
\hline Overload & $\begin{array}{l}\text { Staff given overloaded expectations by resident or other staff/admin, stretched } \\
\text { beyond capacity }\end{array}$ & 2 & 5 \\
\hline Time & References to staff time constraints; Scheduling constraints & 5 & 10 \\
\hline Ratio & $\begin{array}{l}\text { Staff to resident ratio is too low to achieve care; Multiple people at the same } \\
\text { time }\end{array}$ & 3 & 7 \\
\hline Financial Resources & Lack of money (of resident or facility) to do preference & 1 & 2 \\
\hline Government Policy & Department of Health, etc., policies restrict preferences & 1 & 2 \\
\hline Safety & $\begin{array}{l}\text { Safety or health risks restricts preferences (i.e., Wandering, elopement risks, } \\
\text { ulcers) }\end{array}$ & 4 & 8 \\
\hline \multicolumn{4}{|c|}{ Social Environment as Barriers } \\
\hline Lack of Network & $\begin{array}{l}\text { Resident perception of lack of social peer network or lack of network with } \\
\text { matched interests }\end{array}$ & 2 & 5 \\
\hline Contagion & Negative social contagion prevents/restricts preference & 1 & 1 \\
\hline Family & Family preferences differ from residents and affects how can help resident & 6 & 11 \\
\hline Resident Relationships & Resident lack of fit with others, do not get along & 1 & 2 \\
\hline Generations & Gap of generations between residents which makes it hard for staff to please & 1 & 1 \\
\hline Support & Lack of support from administration or other staff as barrier to staff & 2 & 3 \\
\hline Respect & Lack of resident/other staff respect to staff & 1 & 2 \\
\hline Threat & Threats to staff by residents to call authorities, be fired, get in trouble, etc. & 2 & 7 \\
\hline \multicolumn{4}{|c|}{ Resident Characteristics as Barriers } \\
\hline Behaviors & Behaviors of resident restrict preference from being met & 4 & 7 \\
\hline Persistence & Resident very persistent on what/when/why & 2 & 4 \\
\hline Interest ${ }^{* *}$ & $\begin{array}{l}\text { Different interests in activities/preferences, etc., as a barrier to fulfillment, or a } \\
\text { lack of similar interests }\end{array}$ & 2 & 3 \\
\hline Mood $^{* *}$ & Resident mood restricts preference fulfillment & $\overline{2}$ & 2 \\
\hline
\end{tabular}




\begin{tabular}{|c|c|c|c|}
\hline Code Name & Theme Description & Groups $^{a}$ & Citations $b$ \\
\hline Personal Health $^{* *}$ & Physical health affects ability to participate & 4 & 7 \\
\hline Mental Health & Mental health is a barrier to preference being met & 4 & 4 \\
\hline Personal Resources ${ }^{* *}$ & Lack of family resources/support for care (i.e., no family to support care) & 1 & 1 \\
\hline Personal Schedule ${ }^{* *}$ & Resident personal schedule restricts preference fulfillment & 1 & 2 \\
\hline Functional Ability ${ }^{* *}$ & Physical ability of resident, speech, incontinence, etc. & 4 & 11 \\
\hline Sensory Ability ${ }^{* *}$ & Hearing/vision impairment of the resident restricts fulfillment & 2 & 3 \\
\hline Status Change & Change in ability of resident as a barrier to meeting preferences & 2 & 3 \\
\hline Cognition $^{* *}$ & Cognitive ability prevents preferences from being met & 5 & 9 \\
\hline Competency $^{* *}$ & Resident lacks ability to do the task, or thinks lack of ability/defeat & 3 & 6 \\
\hline Past Experience & Resident past negative experience impacts current inability to meet preference & 1 & 1 \\
\hline Social Acceptability ${ }^{* *}$ & Resident embarrassment, or concerns about fitting in & 2 & 2 \\
\hline Choice $^{* * *}$ & Resident choice is restricted which prevents preference from being met & 1 & 1 \\
\hline Education & $\begin{array}{l}\text { Lack of understanding/education of disease/needs/diagnosis by resident or } \\
\text { family }\end{array}$ & 2 & 3 \\
\hline
\end{tabular}

Note. $N=36$ staff members within 6 groups.

**

Denotes barriers that were also listed by residents as barriers to preference fulfillment (see Heid et al., 2014).

${ }^{a}$ Indicates the number of groups (Dietary staff, Resident Care Assistants - Morning shift, Resident Care Assistants - Evening shift, Nursing staff, Recreation staff, and Social Workers) the theme presented for.

$b_{\text {Indicates the number of citations of each theme in the transcripts. }}$ 


\section{Table 5}

Staff Perceived Reasons of why Residents may Change their Minds about their Preferences (i.e., Dependencies)

\begin{tabular}{|c|c|c|c|}
\hline Code Name & Description & Groups $^{a}$ & Citations ${ }^{b}$ \\
\hline \multicolumn{4}{|c|}{ Global Characteristics as Dependencies } \\
\hline Weather $^{* *}$ & Weather changes resident preferences & 1 & 2 \\
\hline \multicolumn{4}{|c|}{ Social Environment as Dependencies } \\
\hline Family Relationships & $\begin{array}{l}\text { Relationship of resident with family members affects preferences; Family pushed for } \\
\text { something that made resident change mind; "Provoked preference" }\end{array}$ & 2 & 3 \\
\hline Resident Relationships & Other residents at activity or involved in activity change person's mind & 2 & 7 \\
\hline Staff Relationships & Relationship of resident with staff doing activity with them & 3 & 6 \\
\hline \multicolumn{4}{|c|}{ Resident Characteristics as Dependencies } \\
\hline Personal Health ${ }^{* * *}$ & How resident is feeling, rested/tired & 4 & 6 \\
\hline Mental Health & Mental illness of resident & 2 & 3 \\
\hline Mood $^{* *}$ & How resident feel emotionally that day/time & 3 & 7 \\
\hline Functional Ability ${ }^{* *}$ & Physical ability of resident & 1 & 2 \\
\hline Sensory Ability ${ }^{* *}$ & Vision/hearing impairment of the resident & 1 & 1 \\
\hline Social Acceptability ${ }^{* *}$ & Resident embarrassment or fitting in; Idealized sense of self & 3 & 3 \\
\hline General Change ${ }^{* *}$ & General changing of one's mind by resident; "It is a woman's prerogative" & 5 & 5 \\
\hline Envy & Residents want what other resident has; "Grass is greener" & 4 & 7 \\
\hline Choice $^{* *}$ & Resident ability to choose or have opportunity; Sense of control of resident & 2 & 2 \\
\hline Cognition $^{* *}$ & $\begin{array}{l}\text { Residents' global cognitive impairment and specifically memory loss creates change in } \\
\text { preferences }\end{array}$ & 5 & 10 \\
\hline Competency $^{* *}$ & Not matched to resident competence level, too high or too low; Success in activity & 2 & 3 \\
\hline Interest $^{* *}$ & Resident interest in type of activity or doing something & 4 & 7 \\
\hline \multicolumn{4}{|c|}{ Other Staff Perception as Dependencies } \\
\hline Kids & Staff reference to residents change of mind because they are like kids & 1 & 1 \\
\hline
\end{tabular}

Note. $N=36$ staff members within 6 groups.

**

Denotes dependencies that were also listed by residents as dependencies to preference fulfillment (see Heid et al., 2014).

andicates the number of groups (Dietary staff, Resident Care Assistants - Morning shift, Resident Care Assistants - Evening shift, Nursing staff, Recreation staff, and Social Workers) the theme presented for.

$b_{\text {Indicates the number of citations of each theme in the transcripts. }}$ 